\title{
Evaluating severity of white matter lesions from computed tomography images with convolutional neural network
}

\author{
Johanna Pitkänen ${ }^{1}$ (D) Juha Koikkalainen ${ }^{2} \cdot$ Tuomas Nieminen $^{2} \cdot$ Ivan Marinkovic ${ }^{1} \cdot$ Sami Curtze $^{1} \cdot$ Gerli Sibolt $^{1}$. \\ Hanna Jokinen ${ }^{1,3}$. Daniel Rueckert ${ }^{4}$. Frederik Barkhof ${ }^{5,6,7} \cdot$ Reinhold Schmidt $^{8} \cdot$ Leonardo Pantoni $^{9}$. \\ Philip Scheltens ${ }^{10} \cdot$ Lars-Olof Wahlund $^{11}$ - Antti Korvenoja ${ }^{12}$ - Jyrki Lötjönen ${ }^{2} \cdot$ Timo Erkinjuntti $^{1} \cdot$ Susanna Melkas $^{1}$
}

Received: 6 November 2019 / Accepted: 24 March 2020 / Published online: 13 April 2020

(C) The Author(s) 2020

\begin{abstract}
Purpose Severity of white matter lesion (WML) is typically evaluated on magnetic resonance images (MRI), yet the more accessible, faster, and less expensive method is computed tomography (CT). Our objective was to study whether WML can be automatically segmented from CT images using a convolutional neural network (CNN). The second aim was to compare CT segmentation with MRI segmentation.

Methods The brain images from the Helsinki University Hospital clinical image archive were systematically screened to make CT-MRI image pairs. Selection criteria for the study were that both CT and MRI images were acquired within 6 weeks. In total, 147 image pairs were included. We used CNN to segment WML from CT images. Training and testing of CNN for CT was performed using 10-fold cross-validation, and the segmentation results were compared with the corresponding segmentations from MRI.

Results A Pearson correlation of 0.94 was obtained between the automatic WML volumes of MRI and CT segmentations. The average Dice similarity index validating the overlap between CT and FLAIR segmentations was 0.68 for the Fazekas 3 group. Conclusion CNN-based segmentation of CT images may provide a means to evaluate the severity of WML and establish a link between CT WML patterns and the current standard MRI-based visual rating scale.
\end{abstract}

Keywords Cerebral small vessel disease $\cdot$ Convolutional neural network $\cdot$ Computed tomography $\cdot$ Machine learning $\cdot$ White matter lesions

Johanna Pitkänen

johanna.pitkanen@hus.fi

1 Department of Neurology, University of Helsinki and Helsinki University Hospital, PO Box 302, 00029 HUS Helsinki, Finland

2 Combinostics Ltd., Tampere, Finland and VTT Technical Research Centre of Finland, Tampere, Finland

3 Department of Psychology and Logopedics, Faculty of Medicine, University of Helsinki, Helsinki, Finland

4 Biomedical Image Analysis Group, Department of Computing, Imperial College London, London, UK

5 Department of Radiology and Nuclear Medicine, Neuroscience Campus Amsterdam, VU University Medical Center, Amsterdam, The Netherlands

6 Institutes of Neurology and Healthcare Engineering, University College London, London, UK
7 NIHR Biomedical Research Centre at University College London Hospitals NHS Foundation Trust and University College London, London, England, UK

8 Department of Neurology, Neuroscience Campus Amsterdam, VU University Medical Center, Amsterdam, Netherlands

9 L. Sacco Department of Biomedical and Clinical Sciences, University of Milan, Milan, Italy

10 Alzheimer Center and Department of Neurology, Neuroscience Campus Amsterdam, VU University Medical Center, Amsterdam, Netherlands

11 Department of Neurobiology, Care Sciences and Society, Division of Clinical Geriatrics, Karolinska Institutet, Stockholm, Sweden

12 HUS Medical Imaging Center, Radiology, University of Helsinki and Helsinki University Hospital, Helsinki, Finland 


\section{Introduction}

White matter lesions (WML) are a surrogate for cerebral small vessel disease (SVD), which is the major cause of accumulating vascular burden in aging populations. Severe WML in stroke patients are associated with a risk of complications after thrombolysis [1] and poor prognosis after carotid endarterectomy [2]. Other well-documented consequences of severe WML are cognitive impairment, gait disturbances, depression, urine incontinence, and the eventual loss of independence and risk for permanent institutionalization [3, 4].

The most common method for grading WML extent has been the Fazekas visual rating scale developed for MRI $[5,6]$. It was preceded by several proposals for CT-based visual rating scales by the authors Gorter [7], Blennow et al. [8], van Swieten et al. [9], and Wahlund et al. [10] which have not been widely adopted in clinical practice $[6,11]$.

Computer-aided image analysis and machine learning methods are increasingly used in medicine. They enable automated and quantitative analyses of large image databases and help to develop tools that complement the manual visual assessment. Advances in machine learning, especially in the field of deep learning, have improved the ability to identify, quantify, and classify patterns in medical images [11].

Deep learning methods, in particular convolutional neural networks (CNNs), have become the state-of-the-art methods for medical image analysis tasks. Modern central processing units (CPUs) and graphics processing units (GPUs) are powerful enough to process large amount of data with advanced learning algorithms [12]. CNNs take a large number of training samples as an input and build a model with a vast number of parameters that will predict the output based on the training examples. CNNs use convolution operation to learn the features such as edges, patterns, and colors from the input images [13]. They have been applied in several image processing tasks such as image segmentation [14] and image classification [15]. Recently, CNNs have also been applied to medical image analysis $[16,17]$.

In this study, the objective was to study if the WML can be automatically segmented from $\mathrm{CT}$ images using CNN. The aim was also to compare CT segmentation with MRI segmentation.

\section{Methods}

\section{Participants and design}

Brain images from the Helsinki University Hospital clinical image archive were systemically screened by qualified healthcare professionals from January 2014 to December 2016 to make CT-MRI image pairs. The images were from the Helsinki University Hospital, and from five area hospitals in the Helsinki region. MRIs were acquired with Siemens and Philips scanners, and CT scanners included Siemens and GE devices.

Thirteen FLAIR images were sagittal 3D images with inplane resolution $0.45-0.47 \mathrm{~mm}$ and slice thickness 0.9 $1.2 \mathrm{~mm}$. The remaining 136 images were $2 \mathrm{D}$ axial images with in-plane resolution $0.43-0.98 \mathrm{~mm}$ and slice thickness $4.0-5.0 \mathrm{~mm}$. The in-plane resolution of CT images was $0.41-1.0 \mathrm{~mm}$, and the slice thickness was $1.0-5.0 \mathrm{~mm}$.

Selection criteria for the study were that both CT and MRI images were acquired and the time interval between $\mathrm{CT}$ and MRI imaging was less than 6 weeks. Images with tumors, cortical infarcts, hematomas (except microbleeds), and multiple sclerosis lesions and contusions were excluded. The images were divided into three Fazekas groups (Fazekas 0-1 = no to mild WML, Fazekas $2=$ moderate WML, Fazekas $3=$ severe WML) according to radiologists' evaluation of the MRI image. The evaluation was made both by general radiologists and neuroradiologists. In total, 147 image pairs were included in the study (Table 1).

Ethical review for retrospective analysis of imaging data collected prospectively as part of routine clinical care is not required at our institution. The analysis of image pairs was anonymized and no clinical data was handled in connection to this analysis.

\section{Automated image analysis}

The analysis pipeline is presented in Fig. 1. The preprocessing steps included skull-stripping, coarse spatial normalization, and coarse intensity normalization of the images. The skull-stripping (brain extraction) of the MRI FLAIR images was performed using the cNeuro® cMRI image quantification tool (Combinostics Ltd., Tampere, Finland). Spatial normalization was performed by registering the binary brain mask to the corresponding brain mask of a mean anatomical template image using 9-degree of freedom affine registration. A CT image was registered with the FLAIR image using rigid registration by maximizing the normalized mutual information. Finally, the intensities were normalized by $z$-scoring within the brain mask.

The resulting pre-processed images were used as inputs in CNN segmentation. Two CNN models were created. (1) CNN for MRI was developed using FLAIR segmentations from the

Table 1 Demographics of the dataset

\begin{tabular}{llcl}
\hline & Mean age & SD age & $\%$ females \\
\hline All $N=147$ & 71.2 & 9.7 & $55 \%$ \\
Fazekas $0-1 N=50$ & 65.7 & 11.5 & $58 \%$ \\
Fazekas $2 N=48$ & 73.4 & 7.1 & $56 \%$ \\
Fazekas $3 N=49$ & 74.7 & 7.2 & $51 \%$ \\
\hline
\end{tabular}


Fig. 1 Flowchart of the analysis $(n=147)$

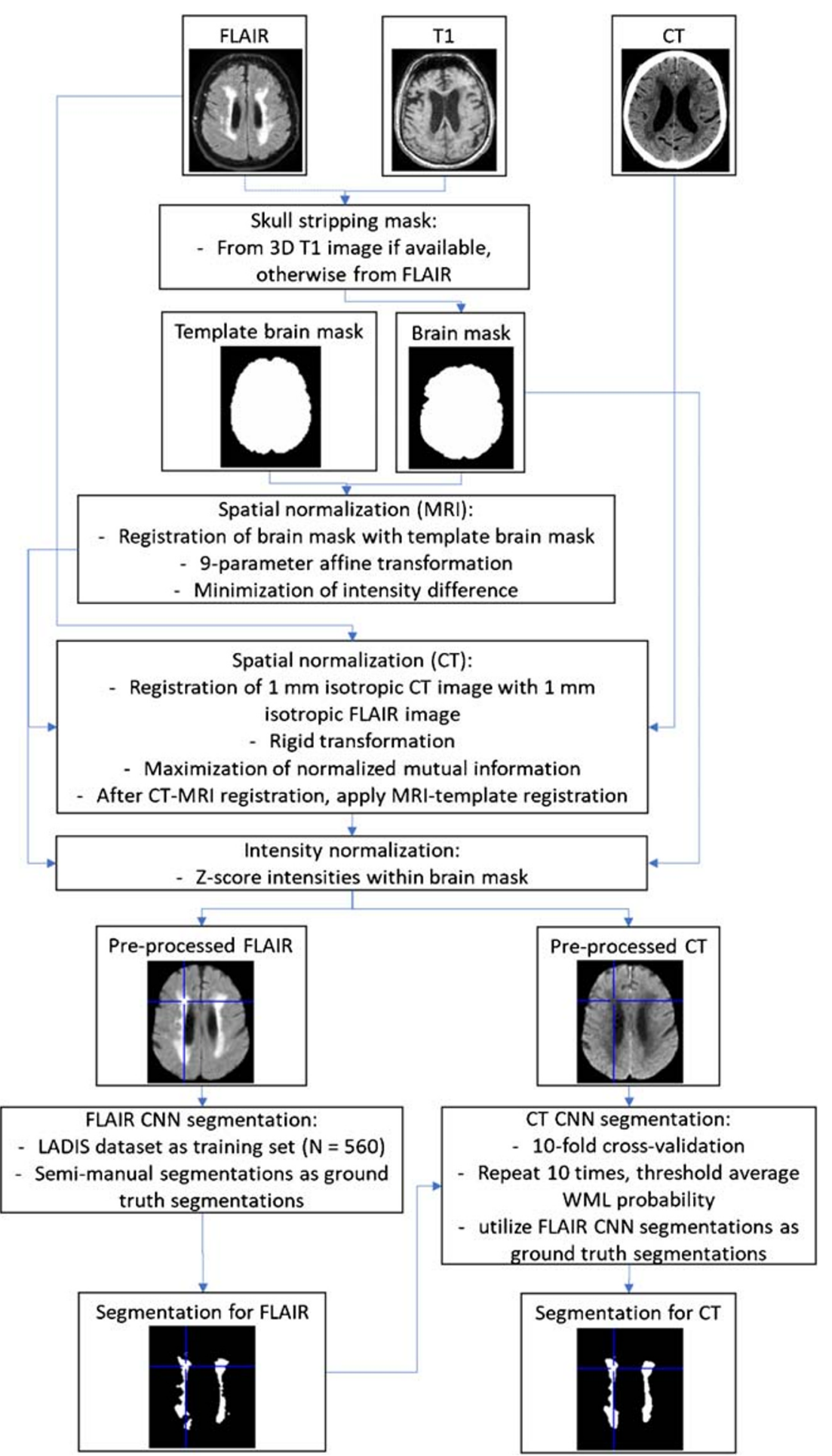

LADIS study (Leukoaraiosis and Disability study) as training data (560 FLAIR images with semi-manually segmented WML). (2) CNN for CT was developed using MRI segmentations from the MRI-CT pairs as training data.

MRI and CT images were segmented using $\mathrm{CNN}$ without and with 10-fold cross-validation, respectively. The CT images were selected randomly so that $90 \%$ of the cases established the training set, and the remaining $10 \%$ of the cases established the test set. This was repeated ten times so that each case was once used in a test set. The WML segmentations of the FLAIR images were used as the ground truth segmentations for training. To improve the robustness of the segmentation, the 10-fold cross-validation was repeated ten times so that ten separate segmentations were obtained for 


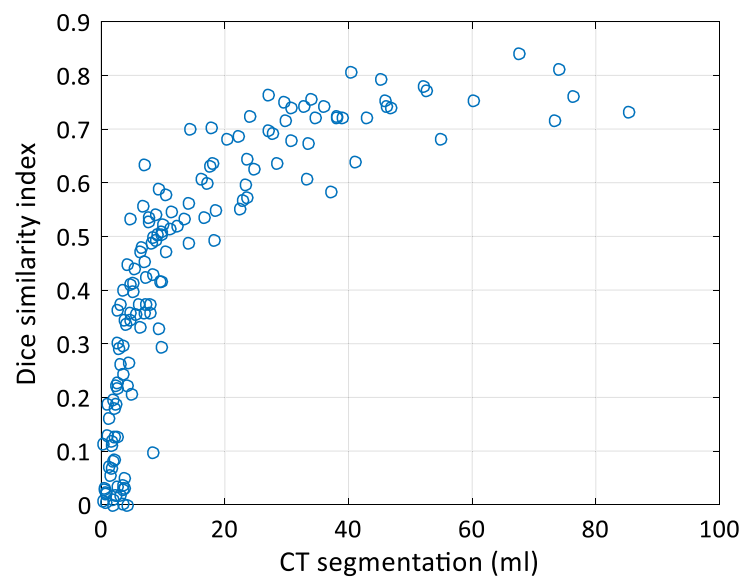

(a)

Fig. 2 The accuracy of the segmentation of CT images. a The Dice similarity index as a function of the WML volume. The distribution of the WML volumes as a function of Fazekas score. b The volumes of

each CT image. The CNN segmentation gives the probability of the WML as an output. The final segmentation was generated by averaging the probabilities of the ten segmentations, and thresholding the average probability using a value of 0.25 .

CNN segmentations (both FLAIR and CT) were performed using U-shaped CNN called uResNet [15]. In this study, we used the network architecture proposed by Guerrero et al. [18] that was originally developed for the segmentation of white matter hyperintensities and stroke lesions from FLAIR images. This network, without any further modifications, was implemented using Theano 0.9.0 (http://www.deeplearning. net/software/theano/) deep learning Python (Python 2.7) library. The $\mathrm{CNN}$ was trained using large image patches $(64 \times 64)$. This allows the network to learn the high- and low-level features from the input images. During the training, CNN parameters were optimized so that the error between the predicted segmentations and "ground truth" segmentations was as small as possible.

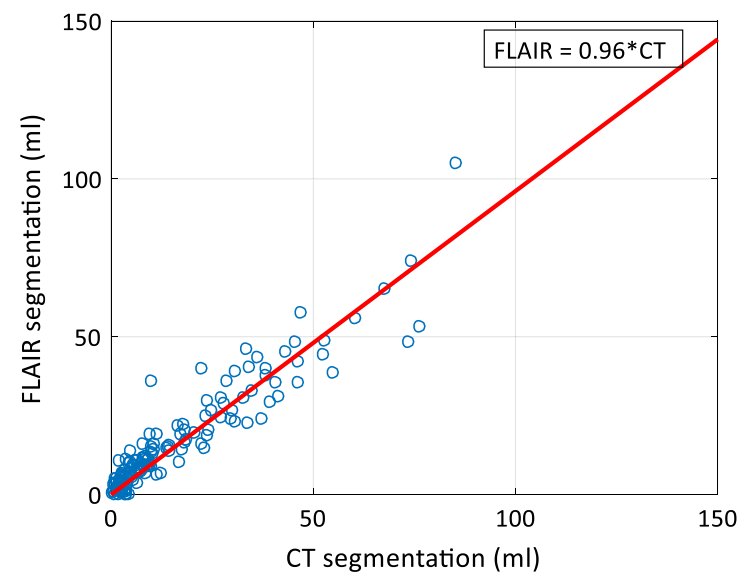

(a)

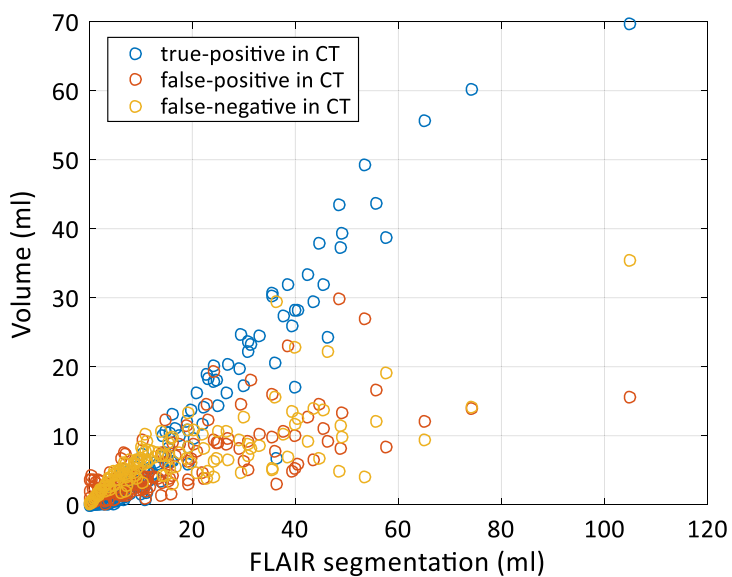

(b)

correctly and incorrectly segmented voxels in CT images as compared with the segmentation of FLAIR images

\section{Statistical analysis}

The accuracy of the CT WML segmentations was validated by comparing the segmentations to the corresponding segmentations from the FLAIR images. The accuracy of the CT segmentations was evaluated by keeping the segmentation of FLAIR images as a ground truth. The Dice overlap measures that the ratio of voxels segmented as WML in both images and the voxels segmented as WML in CT and in FLAIR: Dice $=\frac{2|X \cap Y|}{|X|+|Y|}$, where $|X|$ and $|Y|$ are the WML volumes of the CT and FLAIR segmentations, and $|X \cap Y|$ is the volume of voxels segmented as WML in both CT and FLAIR. In addition, the accuracy of the segmentation was evaluated by studying the volume of correctly and incorrectly segmented voxels. The correlation of the volumes of CT and FLAIR segmentations was evaluated by computing the Pearson correlation. In addition, the Fazekas score was estimated from the

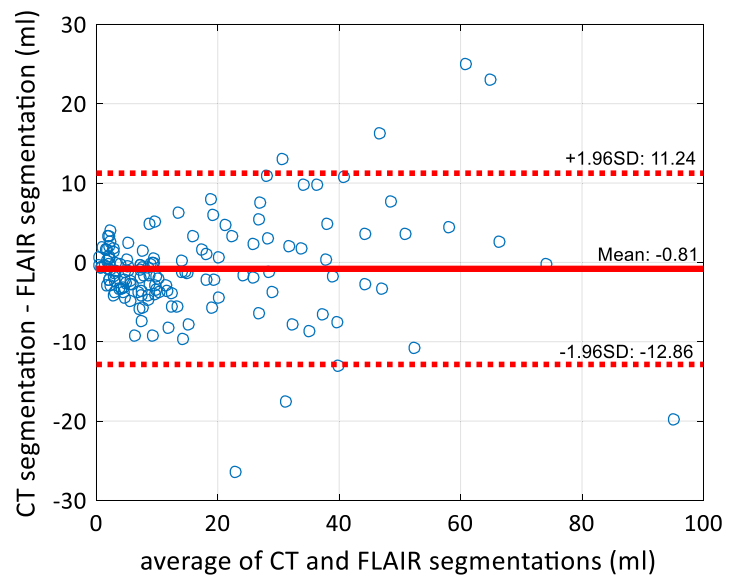

(b)

Fig. 3 The correlation of the volumes. a The correlation between the WML volumes segmented from CT and FLAIR images. The correlation coefficient was 0.94. b The Bland-Altman plot for the differences of CT and FLAIR segmentations 


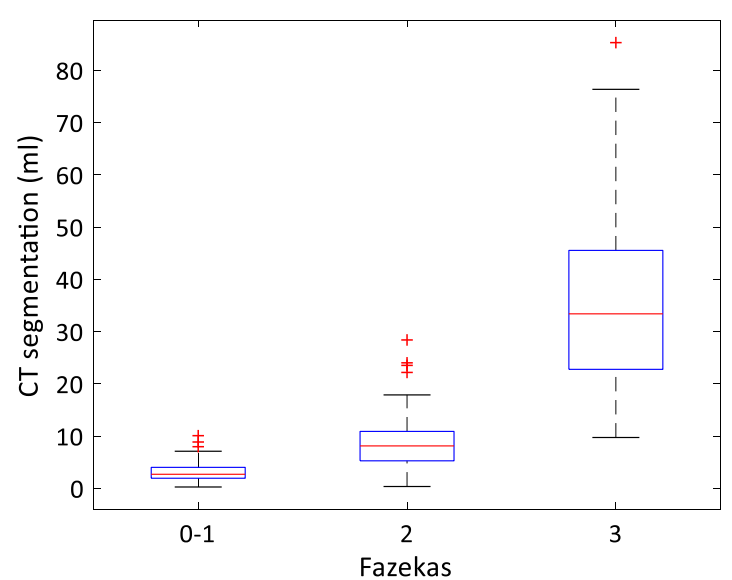

(a)

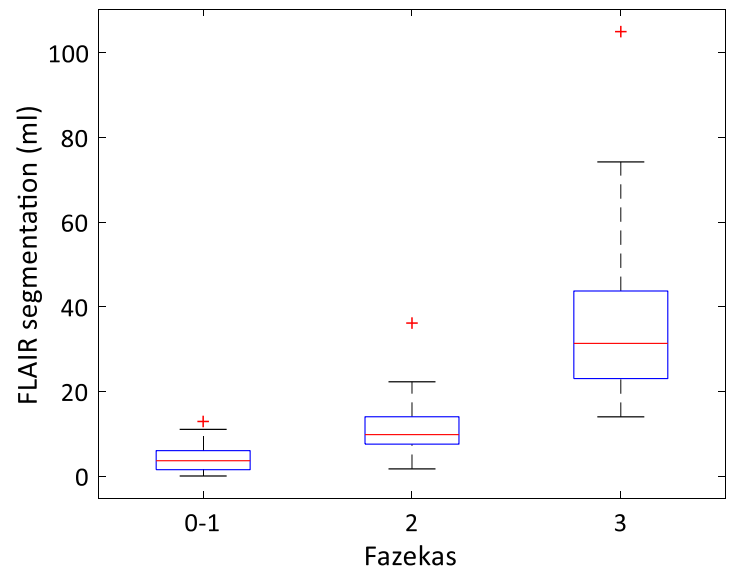

(b)

Fig. 4 The distribution of the WML volumes as a function of Fazekas score $\mathbf{a}$ for CT and $\mathbf{b}$ for FLAIR segmentations

WML volumes by searching the optimal thresholds for the three Fazekas groups used $(0-1,2$, and 3). These computations were performed using 10 -fold cross-validation.

\section{Results}

The Dice similarity index validating the overlap between CT and FLAIR segmentations is presented as the function of the WML volume in Fig. 2a. As expected, the index values are low for small WML volumes: the average Dice similarity index was 0.43 for the whole dataset. However, the more WML there are, the higher values are obtained: the average Dice similarity index value for the Fazekas 3 group was 0.68 . The volumes of correctly segmented voxels, the voxels segmented as WML in CT but as background in FLAIR, and the voxels segmented as WML in FLAIR but as background in CT are presented in Fig. 2 b.

The correlation of the WML volumes of the CT and FLAIR segmentations is shown in Fig. 3a. The volumes of the CT and FLAIR segmentations are strongly correlated (correlation coefficient 0.94 ). Also, the slope of the curve fitted to the data is 0.96 , close to 1 -i.e., the CT segmentation neither underestimates nor

Table 2 Confusion matrix of the estimated Fazekas scores based on the automatic WML volumes using CT (share of correct estimates $=0.78$ ) and FLAIR ( share of correct estimates $=0.78$ )

\begin{tabular}{|c|c|c|c|c|}
\hline \multirow[t]{2}{*}{ CT } & & \multicolumn{3}{|c|}{ Automatic score } \\
\hline & & $0-1$ & 2 & 3 \\
\hline \multirow[t]{3}{*}{ Visual score } & $0-1$ & 43 & 7 & 0 \\
\hline & 2 & 12 & 28 & 8 \\
\hline & 3 & 0 & 5 & 44 \\
\hline \multirow[t]{2}{*}{ FLAIR } & & \multicolumn{3}{|c|}{ Automatic score } \\
\hline & & $0-1$ & 2 & 3 \\
\hline \multirow[t]{3}{*}{ Visual score } & $0-1$ & 37 & 13 & 0 \\
\hline & 2 & 7 & 37 & 4 \\
\hline & 3 & 0 & 9 & 40 \\
\hline
\end{tabular}

overestimates the WML volume as compared with the FLAIR segmentation, which can be seen also in the Bland-Altman plot in Fig. $3 b$.

The distributions of the WML volumes for different Fazekas groups for CT and FLAIR segmentations are shown in Fig. 4 a and $b$, respectively. The Fazekas groups have clearly distinct distributions in both cases, and qualitatively, the CT and FLAIR distributions are very similar. Table 2 presents the results for estimating the Fazekas scores from the WML volumes when compared with the ground truth visual ratings. The score was correctly estimated in $78 \%$ of cases from both CT and FLAIR images.

Figure 5 shows the example segmentations for the CT and FLAIR images of patients from each Fazekas group. This shows that especially when the WML volume is high, the CT segmentation is able to produce corresponding results with the FLAIR segmentation.

\section{Discussion}

Our study suggests that the severity of WML can be estimated from CT images, using automated image analysis methods, with results very similar to those using the Fazekas scale for MRI images. These methods provide means for volumetric assessment of the burden of WML as an alternative to visual scaling. There was a high correlation of 0.94 obtained between the automatic WML volumes of MRI and CT segmentations. The ratings produced automatically both from CT and MRI were equal to visual ratings in $78 \%$ of the cases.

Previously, an automated method for quantifying CT cerebral WML has been under evaluation in a multicenter validation study in the UK [19]. The automated WML volume correlation at MR imaging was 0.85 and at CT imaging 0.71 when compared with expert-delineated WML volumes. The study sample in UK was acute ischemic stroke cases.

In general, the constraint of utilizing CT instead of MRI is the impaired detection of small lesions including punctate and early confluent changes. However, CT seems to be sufficient when 


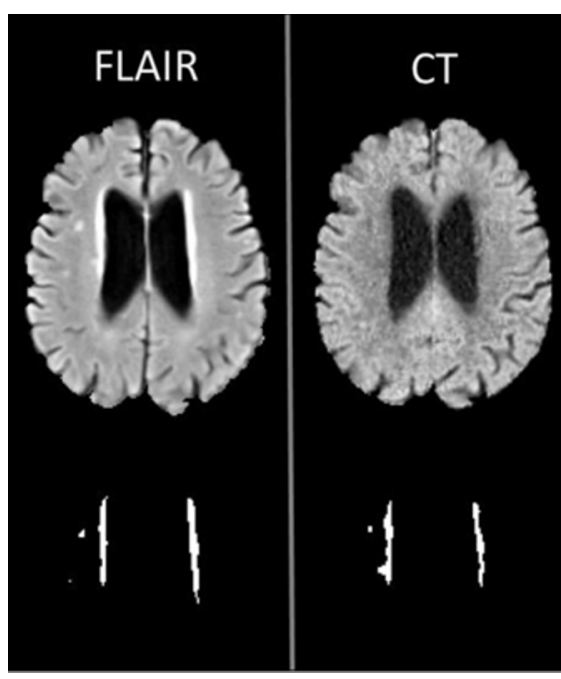

Fazekas 1

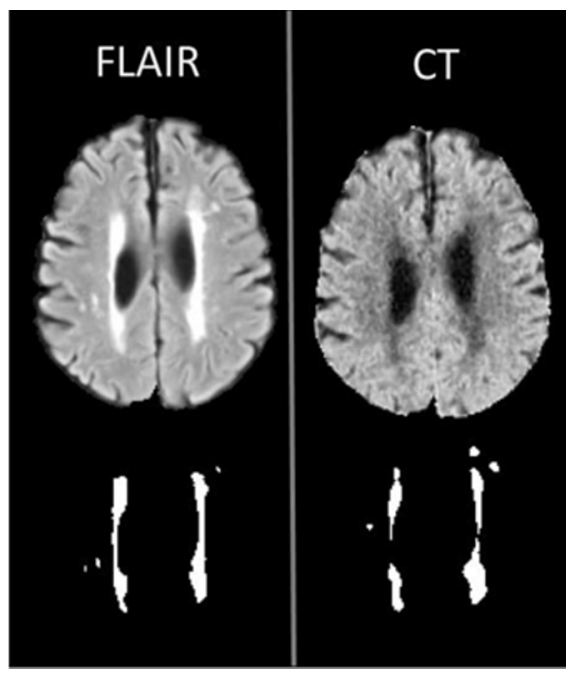

Fazekas 2

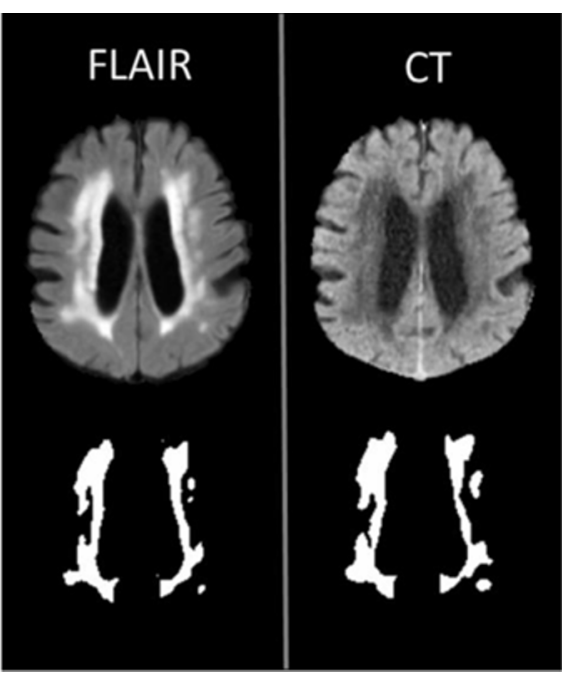

Fazekas 3

Fig. 5 Examples of the FLAIR and CT WML segmentations for the three Fazekas groups

using a multi-detector CT with coronal and sagittal reformats [20]. CT is also often used in dementia imaging in clinical practice [21]. Among acute stroke patients, non-contrast CT is the most common initial imaging modality in clinical practice [22]. From the clinical point of view, detecting moderate and severe WML is more relevant than detecting early phase WML (pre-mild or mild), because acute clinical complications and risks are associated with moderate and severe WML [1, 4, 23]. Early phase WML is more relevant in younger age groups participating in follow-up and in intervention studies. In this setting, the patients are more likely to undergo MRI.

We used the automated FLAIR WML segmentations from the LADIS cohort as the ground truth segmentations when training the CNN model and validating the CT segmentation results. Our previous cross-validated study [24] has shown that the CNNbased WML segmentation on MRIs produces very similar results when compared with the semi-manual segmentation (correlation 0.99 , average Dice similarity index 0.72). This suggests that the MRI-based CNN WML segmentation can be used as ground truth in training CT CNN models and can also be used in validation.

A relatively small dataset is a limitation in this study, and a totally independent validation set is needed in future studies. It is possible that our findings are in some extent obscured by the presence of lacunes, but probably this influence is of minor importance because lacunes have a distinct morphology that does not confuse with WML. Microbleeds were not regarded because they are invisible on CT. In the present study, patients with concomitant lesions like cortical infarcts or tumor edema were excluded, which is a limitation. In future studies, such combined lesions could be evaluated with deep learning requiring a larger training set with good representation of different lesion types. Also, the lack of clinical data is a limitation in our study.

The strength of our study is that the images were unselected. The patients were not exclusively stroke patients nor other neurological patients. The CT and MRI equipment as well as the imaging parameters varied. Therefore, the results and the models are more likely to generalize to other datasets. While this will likely increase variability in segmentation results, we consider that the scanner differences and different scan parameters (such as different $\mathrm{kV}$ or double energy) do not affect the results as far as the analysis is restricted to moderate or severe WML. The influence of different variables on variance could be analyzed in future studies.

Automated volumetric rating could direct radiologists towards a uniform evaluation of WML and might increase clinician's alertness for WML and its influences on treatment and outcomes. Automated rating enables a variety of analyses in cohorts of stroke patients and other neurological patients and studies can be cross-evaluated worldwide. In the future, it will be interesting to study the correlation with clinical data to see if CT segmentation leads to similar results in terms of clinical correlation when compared with MRI segmentation. Although the present study suggests a clinical solution, the method is still not all-inclusive and thus calls for further research, for example, for segmentation in Fazekas grades 1 and 2. More uniform imaging parameters will likely aid in achieving this goal.

Acknowledgements Open access funding provided by University of Helsinki including Helsinki University Central Hospital.

Funding information This study was funded by grants (Y1249NEUR2) from Department of Neurology, Helsinki University Hospital and (TYH2016207) State funding for university-level health research.

\section{Compliance with ethical standards}

Conflict of interest The authors Juha Koikkalainen and Jyrki Lötjönen are shareholders of Combinostics Ltd. All the other authors declare that they have no conflict of interest.

Ethical approval For this type of the study, formal consent is not required. Ethical review for retrospective analysis of imaging data collected 
prospectively as part of routine clinical care is not required at our institution.

Open Access This article is licensed under a Creative Commons Attribution 4.0 International License, which permits use, sharing, adaptation, distribution and reproduction in any medium or format, as long as you give appropriate credit to the original author(s) and the source, provide a link to the Creative Commons licence, and indicate if changes were made. The images or other third party material in this article are included in the article's Creative Commons licence, unless indicated otherwise in a credit line to the material. If material is not included in the article's Creative Commons licence and your intended use is not permitted by statutory regulation or exceeds the permitted use, you will need to obtain permission directly from the copyright holder. To view a copy of this licence, visit http://creativecommons.org/licenses/by/4.0/.

\section{References}

1. Curtze S, Melkas S, Sibolt G et al (2015) Cerebral computed tomography-graded white matter lesions are associated with worse outcome after thrombolysis in patients with stroke. Stroke 46: $1554-1560$

2. Oksala N, Jaroma M, Pienimaki JP et al (2014) Preoperative white matter lesions are independent predictors of long-term survival after internal carotid endarterectomy. Cerebrovasc Dis Extra 4:122-131

3. The LADIS Study Group, Poggesi A, Pantoni L et al (2011) 20012011: a decade of the LADIS (leukoaraiosis and DISability) study: what have we learned about white matter changes and small-vessel disease? Cerebrovasc Dis 32:577-588

4. Sibolt G, Curtze S, Melkas S, Pohjasvaara T, Kaste M, Karhunen PJ, Oksala NK, Erkinjuntti T (2015) Severe cerebral white matter lesions in ischemic stroke patients are associated with less time spent at home and early institutionalization. Int J Stroke 10:11921196

5. Pantoni L, Basile AM, Pracucci G, Asplund K, Bogousslavsky J, Chabriat H, Erkinjuntti T, Fazekas F, Ferro JM, Hennerici M, O'brien J, Scheltens P, Visser MC, Wahlund LO, Waldemar G, Wallin A, Inzitari D (2005) Impact of age-related cerebral white matter changes on the transition to disability-the LADIS study: rationale, design and methodology. Neuroepidemiology 24(1-2): 51-62

6. Wardlaw JM, Smith EE, Biessels GJ et al (2013) Neuroimaging standards for research into small vessel disease and its contribution to ageing and neurodegeneration. Lancet Neurol 12:822-838

7. Gorter JW (1999) Major bleeding during anticoagulation after cerebral ischemia: patterns and risk factors. Stroke Prevention In Reversible Ischemia Trial (SPIRIT). European Atrial Fibrillation Trial (EAFT) study groups. Neurology 53:1319-1327

8. Blennow K, Wallin A, Uhlemann C, Gottfries CG (1991) Whitematter lesions on $\mathrm{CT}$ in Alzheimer patients: relation to clinical symptomatology and vascular factors. Acta Neurol Scand 83: 187-193

9. van Swieten JC, Hijdra A, Koudstaal PJ, van Gijn J (1990) Grading white matter lesions on CT and MRI: a simple scale. J Neurol Neurosurg Psychiatry 53:1080-1083

10. Wahlund LO, Barkhof F, Fazekas F, Bronge L, Augustin M, Sjögren M, Wallin A, Ader H, Leys D, Pantoni L, Pasquier F, Erkinjuntti T, Scheltens P, European Task Force on Age-Related White Matter Changes (2001) A new rating scale for age-related white matter changes applicable to MRI and CT. Stroke 32:13181322

11. Shen D, Wu G, Suk HI (2017) Deep learning in medical image analysis. Annu Rev Biomed Eng 19:221-248

12. Litjens G, Kooi T, Bejnordi BE, Setio AAA, Ciompi F, Ghafoorian M, van der Laak J, van Ginneken B, Sánchez CI (2017) A survey on deep learning in medical image analysis. Med Image Anal 42:60-88

13. Goodfellow I, Bengio Y, Courville A (2016) Deep learning, vol 1. MIT press, Cambridge

14. Long J, Shelhamer E and Darrell T (2015) Fully convolutional networks for semantic segmentation. Presented in Proceedings of the IEEE Conference on Computer Vision and Pattern Recognition, pp 3431-3440

15. Krizhevsky A, Sutskever I and Hinton GE (2012) Imagenet classification with deep convolutional neural networks. Advances in neural information processing systems. In: Pereira F et al (ed) NIPS'12 Proceedings of the 25th International Conference on Neural Information Processing Systems - Volume 1.Curran Associates Inc., USA, pp 1097-1105

16. Kamnitsas K, Ledig C, Newcombe VFJ, Simpson JP, Kane AD, Menon DK, Rueckert D, Glocker B (2017) Efficient multi-scale 3D CNN with fully connected CRF for accurate brain lesion segmentation. Med Image Anal 36:61-78

17. Li Q, Cai W, Wand X, Zhou Y, Feng DD and Chen M (2014) Medical image classification with convolutional neural network. In: 13th International Conference on Control Automation Robotics \& Vision (ICARCV), pp 844-848

18. Guerrero R, Qin C, Oktay O et al (2017) White matter hyperintensity and stroke lesion segmentation and differentiation using convolutional neural networks. Neuroimage Clin 17:918934

19. Chen L, Carlton Jones AL, Mair G, Patel R, Gontsarova A, Ganesalingam J, Math N, Dawson A, Aweid B, Cohen D, Mehta A, Wardlaw J, Rueckert D, Bentley P, IST-3 Collaborative Group (2018) Rapid automated quantification of cerebral leukoaraiosis on CT images: a multicenter validation study. Radiology 288:573-581

20. Wattjes M, Henneman W, van der Flier W et al (2009) Diagnostic imaging of patients in a memory clinic: comparison of MR imaging and 64-detector row CT. Radiology Oct 253(1):174-183

21. Vernooij MW, Pizzini FB, Schmidt R et al (2019) Dementia imaging in clinical practice: a European-wide survey of 193 centres and conclusions by the ESNR working group. Neuroradiology 61:633

22. Sanossian N, Fu KA, Liebeskind DS et al (2017) Utilization of emergent neuroimaging for thrombolysis-eligible stroke patients. J Neuroimaging 27:59-64

23. Curtze S, Haapaniemi E, Melkas S, Mustanoja S, Putaala J, Sairanen T, Sibolt G, Tiainen M, Tatlisumak T, Strbian D (2015) White matter lesions double the risk of post-thrombolytic intracerebral hemorrhage. Stroke 46:2149-2155

24. Jokinen H, Koikkalainen J, Laakso HM, Melkas S, Nieminen T, Brander A, Korvenoja A, Rueckert D, Barkhof F, Scheltens P, Schmidt R, Fazekas F, Madureira S, Verdelho A, Wallin A, Wahlund LO, Waldemar G, Chabriat H, Hennerici M, O'Brien J, Inzitari D, Lötjönen J, Pantoni L, Erkinjuntti T (2020) Global burden of small vessel disease-related brain changes on MRI predicts cognitive and functional decline. Stroke 51:170-178

Publisher's note Springer Nature remains neutral with regard to jurisdictional claims in published maps and institutional affiliations. 